\title{
Superluminal pions in a hadronic fluid
}

\author{
Neven Bilić*and Hrvoje Nikolić \\ Rudjer Bošković Institute, \\ P.O. Box 180, 10002 Zagreb, Croatia
}

March 9, 2019

\begin{abstract}
We study the propagation of pions at finite temperature and finite chemical potential in the framework of the linear sigma model with 2 quark flavors and $N_{c}$ colors. The velocity of massless pions in general differs from that of light. One-loop calculations show that in the chiral symmetry broken phase pions, under certain conditions, propagate faster than light.
\end{abstract}

\section{Introduction}

The linear sigma model, originally proposed as a model for strong nuclear interactions [1], today serves as an effective model for the low-energy (low-temperature) phase of quantum chromodynamics. The model exhibits spontaneous breaking of chiral symmetry and restoration at finite temperature. In the chiral symmetry broken phase at zero temperature pions, being massless, propagate with the velocity of light. At finite temperature one would expect chiral pions to propagate slower than light, owing to medium effects. Indeed, this expectation has been confirmed by one-loop calculations in the linear sigma model with no fermions [2, 3, 4].

In this paper we discuss the propagation of pions at nonzero temperature and nonzero finite baryon density in a model with two quark flavors. It turns out that pions in the presence of fermions become superluminal in a certain range of temperature and baryon chemical potential.

A superluminal propagation has recently been studied in the context of the Casimir effect in quantum electrodynamics. Scharnhorst has demonstrated [5] that, when vacuum fluctuations of the electromagnetic field obey periodic boundary conditions in one spatial dimension (corresponding to parallel Casimir plates), then the two-loop corrections to the photon polarization tensor lead to a superluminal propagation of photons. A similarity between the effects of Casimir plates and that of finite temperature on the propagation of photons has been discussed [6], and more general conditions that lead to a superluminal propagation of photons have also been identified.

\footnotetext{
*E-mail: bilic@thphys.irb.hr
} 
A similar effect has been found for photons interacting with fermions [7]. It has been shown that the transverse photons coupled to fermions obeying periodic boundary conditions are faster than light when propagating perpendicularly and are slower than light when propagating parallelly to the compactified dimenson. Since our fermions obey the usual antiperiodic boundary conditions in the compact temporal dimension, one would expect no superluminal effects. However, we demonstrate that in a certain region of temperature and chemical potential below the symmetry restoration point, massles pions propagate faster than light. Moreover, if one of the spatial dimensions is compactified, pions will propagate superluminally or subluminally, depending on the size of the compact dimension and on the boundary conditions.

We organize the paper as follows. In Sec. 2 we describe the model. In Sec. 3 we calculate the dependence of the pion velocity on temperature and chemical potential. We present the results and discussion in Sec. 4. In the concluding section, Sec. 5, we summarize our results.

\section{Effective Lagrangian}

Consider the linear sigma model at finite temperature and finite baryon density. The thermal bath provides a medium which may be assumed to have a homogeneous velocity field. The dynamics of mesons in such a medium is described by an effective chirally symmetric Lagrangian of the form

$$
\begin{aligned}
\mathcal{L}= & \frac{1}{2}\left(a \eta^{\mu \nu}+b u^{\mu} u^{\nu}\right)\left(\partial_{\mu} \sigma \partial_{\nu} \sigma+\partial_{\mu} \boldsymbol{\pi} \partial_{\nu} \boldsymbol{\pi}\right)-\frac{m_{0}^{2}}{2}\left(\sigma^{2}+\boldsymbol{\pi}^{2}\right)-\frac{\lambda}{4}\left(\sigma^{2}+\boldsymbol{\pi}^{2}\right)^{2} \\
& +\bar{\psi}\left(c i \gamma^{\mu} \partial_{\mu}+d i u^{\mu} \partial_{\mu}+\mu u^{\mu} \gamma_{\mu}+g\left(\sigma+i \boldsymbol{\tau} \boldsymbol{\pi} \gamma_{5}\right)\right) \psi
\end{aligned}
$$

where $u_{\mu}$ is the velocity of the fluid, and $\eta_{\mu \nu}$ is the flat Lorentzian metric tensor. The right and left fermions, $\psi_{R}=\frac{1}{2}\left(1+\gamma_{5}\right) \psi, \psi_{L}=\frac{1}{2}\left(1-\gamma_{5}\right) \psi$, are assumed to constitute, respectively, the $\left(\frac{1}{2}, 0\right)$ and $\left(0, \frac{1}{2}\right)$ representation of the chiral $\mathrm{SU}(2) \times \mathrm{SU}(2)$, whereas the mesons $(\sigma, \boldsymbol{\pi})$ belong to the $\left(\frac{1}{2}, \frac{1}{2}\right)$ representation. In the original sigma model [1] the fermion field was a nucleon. We consider fermions to be constituent quarks [8, 9, 10, 11] with additional $N_{c}$ degrees of freedom, "colors", from the $\mathrm{SU}\left(N_{c}\right)$ local gauge group of an underlying gauge theory (QCD). The parameters $a, b, c$, and $d$ depend on the temperature $T$, the chemical potential $\mu$, and the parameters of the model $m_{0}, \lambda$, and $g$, and may be calculated in perturbation theory. At $T=\mu=0$ the medium is absent and $a=c=1$ and $b=d=0$.

If $m_{0}^{2}<0$, chiral symmetry will be spontaneously broken. At the classical level, the $\sigma$ and $\pi$ fields develop nonvanishing expectation values such that

$$
\langle\sigma\rangle^{2}+\langle\boldsymbol{\pi}\rangle^{2}=-\frac{m_{0}^{2}}{\lambda} \equiv f_{\pi}^{2}
$$

It is convenient here to choose

$$
\left\langle\pi_{i}\right\rangle=0, \quad\langle\sigma\rangle=f_{\pi} .
$$

At nonzero temperature the expectation value $\langle\sigma\rangle$ depends on the temperature and vanishes at the chiral transition point. Redefining the fields as

$$
(\sigma, \boldsymbol{\pi}) \rightarrow(\sigma, \boldsymbol{\pi})+\left(\sigma^{\prime}(x), \boldsymbol{\pi}^{\prime}(x)\right),
$$


where $\boldsymbol{\pi}^{\prime}$ and $\sigma^{\prime}$ are quantum fluctuations around the constant values $\boldsymbol{\pi}=\mathbf{0}$ and $\sigma=\langle\sigma\rangle$, respectively, we obtain the effective Lagrangian in which chiral symmetry is explicitly broken:

$$
\begin{aligned}
\mathcal{L}^{\prime}= & \frac{1}{2}\left(a g^{\mu \nu}+b u^{\mu} u^{\nu}\right)\left(\partial_{\mu} \sigma^{\prime} \partial_{\nu} \sigma^{\prime}+\partial_{\mu} \boldsymbol{\pi}^{\prime} \partial_{\nu} \boldsymbol{\pi}^{\prime}\right)-\frac{m_{\sigma}^{2}}{2} \sigma^{\prime 2}-\frac{m_{\pi}^{2}}{2} \boldsymbol{\pi}^{\prime 2}-g^{\prime} \sigma^{\prime}\left(\sigma^{\prime 2}+\boldsymbol{\pi}^{\prime 2}\right) \\
& -\frac{\lambda}{4}\left(\sigma^{\prime 2}+\boldsymbol{\pi}^{\prime 2}\right)^{2}+\bar{\psi}\left(c i \gamma^{\mu} \partial_{\mu}+d i u^{\mu} \partial_{\mu}+\mu u^{\mu} \gamma_{\mu}+g\left(\sigma^{\prime}+i \boldsymbol{\tau} \boldsymbol{\pi}^{\prime} \gamma_{5}\right)\right) \psi .
\end{aligned}
$$

The effective masses and the trilinear coupling $g^{\prime}$ are functions of $\sigma$ defined as

$$
\begin{aligned}
& m_{\sigma}^{2}=m_{0}^{2}+3 \lambda \sigma^{2}, \quad m_{F}=g \sigma \\
& m_{\pi}^{2}=m_{0}^{2}+\lambda \sigma^{2}, \quad g^{\prime}=\lambda \sigma .
\end{aligned}
$$

At temperatures below the chiral transition point the fermion mass remains as in (6) and the meson masses are given by

$$
m_{\pi}^{2}=0 ; \quad m_{\sigma}^{2}=2 \lambda \sigma^{2}
$$

in agreement with the Goldstone theorem. The temperature dependence of the chiral condensate $\sigma$ is obtained by minimizing the thermodynamic potential $\Omega=-(T / V) \ln Z$ with respect to $\sigma$ at fixed inverse temperature $\beta$. At one-loop order, the extremum condition reads 11

$$
\begin{aligned}
\sigma^{2}= & f_{\pi}^{2}-\frac{8 g^{2}}{\lambda} N_{c} \int \frac{d^{3} p}{(2 \pi)^{3}} \frac{1}{2 \omega_{F}} n_{F}\left(\omega_{F}\right) \\
& -3 \int \frac{d^{3} p}{(2 \pi)^{3}} \frac{1}{\omega_{\sigma}} n_{B}\left(\omega_{\sigma}\right)-3 \int \frac{d^{3} p}{(2 \pi)^{3}} \frac{1}{\omega_{\pi}} n_{B}\left(\omega_{\pi}\right),
\end{aligned}
$$

where

$$
\begin{gathered}
\omega_{\pi}=|\boldsymbol{p}| ; \quad \omega_{\sigma}=\left(\boldsymbol{p}^{2}+m_{\sigma}^{2}\right)^{1 / 2}, ; \quad \omega_{F}=\left(\boldsymbol{p}^{2}+m_{F}^{2}\right)^{1 / 2}, \\
n_{F}(\omega)=\frac{1}{e^{\beta(\omega-\mu)}+1}+\frac{1}{e^{\beta(\omega+\mu)}+1}, \\
n_{B}(\omega)=\frac{1}{e^{\beta \omega}-1} .
\end{gathered}
$$

The right-hand side of (8) depends on $\sigma$ through the mass $m_{\sigma}$ given by (77). Solutions to (8) are implicit functions of $T$ and $\mu$. These equations have been derived from the thermodynamic potential in which the loop corrections have been neglected. This approximation corresponds to the leading order in the $1 / N$ expansion, where $N$ is the number of scalar fields 12 . In our case, $N=4$.

\section{Pion velocity}

The propagation of pions is governed by the equation of motion

$$
\partial_{\mu}\left[\left(a \eta^{\mu \nu}+b u^{\mu} u^{\nu}\right)\right] \partial_{\nu} \boldsymbol{\pi}+V(\sigma, \boldsymbol{\pi}, \psi) \boldsymbol{\pi}=0,
$$


where $V$ is the interaction potential the form of which is irrelevant to our consideration.

In the simplest case of a homogeneous flow, Eq. (12) reduces to the wave equation

$$
\left(\partial_{t}^{2}-v^{2} \Delta+\frac{v^{2}}{a} V\right) \boldsymbol{\pi}=0
$$

where the quantity $v$ is the pion velocity given by

$$
v^{2}=\left(1+\frac{b}{a}\right)^{-1} \text {. }
$$

As we shall shortly demonstrate, the constants $a$ and $b$ may be derived from the finitetemperature perturbation expansion of the pion self-energy.

The pion velocity in a sigma model at finite temperature has been calculated at oneloop level by Pisarski and Tytgat in the low-temperature approximation [2] and by Son and Stephanov for temperatures close to the chiral transition point [3, 4]. It has been found that the pion velocity vanishes as one approaches the critical temperature. Here we analyze the whole range of temperatures in the chiral symmetry broken phase.

Consider the pion self-energy $\Sigma(q, T)$ in the limit when the external momentum $q$ approaches 0 . The renormalized inverse pion propagator may, in the limit $q \rightarrow 0$, be expressed in the form

$$
Z_{\pi} \Delta^{-1}=q^{\mu} q_{\mu}-\left.\frac{1}{2 !} q^{\mu} q^{\nu} \frac{\partial}{\partial q^{\mu}} \frac{\partial}{\partial q^{\nu}}(\Sigma(q, T)-\Sigma(q, 0))\right|_{q=0}+\ldots
$$

where the ellipsis denotes the terms of higher order in $q^{\mu}$. The $q^{\mu}$ independent term of the self-energy absorbs in the renormalized pion mass, which is equal to zero in the chiral symmetry broken phase. The subtracted $\mathrm{T}=0$ term has been absorbed in the wave function renormalization factor $Z_{\pi}$. By comparing this equation with the inverse pion propagator derived directly from the effective Lagrangian (15)

$$
\Delta^{-1}=(a+b) q_{0}^{2}-a \boldsymbol{q}^{2}
$$

we can express the parameters $a$ and $b$, and hence the pion velocity, in terms of the second derivatives of $\Sigma(q, T)$ evaluated at $q^{\mu}=0$. At one-loop level the diagrams that give a nontrivial $q$-dependence of $\Sigma$ are the bubble diagrams. Subtracting the $\mathrm{T}=0$ term one finds

$$
\Sigma(q) \equiv \Sigma(q, T)-\Sigma(q, 0)=\Sigma_{B}+\Sigma_{F}
$$

with the contribution of the bosonic and fermionic loops given by

$$
\begin{aligned}
\Sigma_{B}(q)= & -4 g^{2} \int \frac{d^{3} p}{(2 \pi)^{3}} \frac{1}{2 \omega_{\pi} 2 \omega_{\sigma, q}} \\
& \times\left\{\left[n_{B}\left(\omega_{\pi}\right)+n_{B}\left(\omega_{\sigma, q}\right)\right]\left(\frac{1}{\omega_{\sigma, q}+\omega_{\pi}-q_{0}}+\frac{1}{\omega_{\sigma, q}+\omega_{\pi}+q_{0}}\right)\right. \\
& \left.+\left[n_{B}\left(\omega_{\pi}\right)-n_{B}\left(\omega_{\sigma, q}\right)\right]\left(\frac{1}{\omega_{\sigma, q}-\omega_{\pi}-q_{0}}+\frac{1}{\omega_{\sigma, q}-\omega_{\pi}+q_{0}}\right)\right\}
\end{aligned}
$$




$$
\begin{aligned}
\Sigma_{F}(q)= & -8 N_{c} g^{2} \int \frac{d^{3} p}{(2 \pi)^{3}} \frac{1}{2 \omega_{F} 2 \omega_{F, q}} \\
& \times\left\{\frac{-q_{0} \omega_{F, q}+\boldsymbol{q}(\boldsymbol{p}+\boldsymbol{q})}{e^{\beta\left(\omega_{F, q}-\mu\right)}+1}\left(\frac{1}{\omega_{F}+\omega_{F, q}-q_{0}}+\frac{1}{\omega_{F}-\omega_{F, q}+q_{0}}\right)\right. \\
& +\frac{-q_{0}^{2}-q_{0} \omega_{F}+\boldsymbol{q}(\boldsymbol{p}+\boldsymbol{q})}{e^{\beta\left(\omega_{F}-\mu\right)}+1}\left(\frac{1}{\omega_{F, q}+\omega_{F}+q_{0}}+\frac{1}{\omega_{F, q}-\omega_{F}-q_{0}}\right) \\
& +\frac{q_{0} \omega_{F, q}+\boldsymbol{q}(\boldsymbol{p}+\boldsymbol{q})}{e^{\beta\left(\omega_{F, q}+\mu\right)}+1}\left(\frac{1}{\omega_{F}+\omega_{F, q}+q_{0}}+\frac{1}{\omega_{F}-\omega_{F, q}-q_{0}}\right) \\
& \left.+\frac{-q_{0}^{2}+q_{0} \omega_{F}+\boldsymbol{q}(\boldsymbol{p}+\boldsymbol{q})}{e^{\beta\left(\omega_{F}+\mu\right)}+1}\left(\frac{1}{\omega_{F, q}+\omega_{F}-q_{0}}+\frac{1}{\omega_{F, q}-\omega_{F}+q_{0}}\right)\right\},
\end{aligned}
$$

where $\omega_{\sigma, q}=\left[(\boldsymbol{p}-\boldsymbol{q})^{2}+m_{\sigma}^{2}\right]^{1 / 2}, \omega_{F, q}=\left[(\boldsymbol{p}+\boldsymbol{q})^{2}+m_{F}^{2}\right]^{1 / 2}$. A straightforward evaluation of the second derivatives of $\Sigma(q)$ at $q_{\mu}=0$ yields

$$
\begin{gathered}
a=1+a_{B}+a_{F}, \\
b=b_{B}+b_{F},
\end{gathered}
$$

with

$$
\begin{gathered}
a_{B}=\frac{16 g^{\prime 2}}{m_{\sigma}^{4}} \int \frac{d^{3} p}{(2 \pi)^{3}}\left[\frac{n_{B}\left(\omega_{\pi}\right)}{4 \omega_{\pi}}+\frac{n_{B}\left(\omega_{\sigma}\right)}{4 \omega_{\sigma}}-\frac{1}{3} \frac{\omega_{\pi}^{2}}{m_{\sigma}^{2}}\left(\frac{n_{B}\left(\omega_{\pi}\right)}{\omega_{\pi}}-\frac{n_{B}\left(\omega_{\sigma}\right)}{\omega_{\sigma}}\right)\right] \\
b_{B}=\frac{16 g^{\prime 2}}{m_{\sigma}^{4}} \int \frac{d^{3} p}{(2 \pi)^{3}}\left[\frac{\omega_{\pi} n_{B}\left(\omega_{\pi}\right)}{m_{\sigma}^{2}}-\frac{\omega_{\sigma} n_{B}\left(\omega_{\sigma}\right)}{m_{\sigma}^{2}}+\frac{1}{3} \frac{\omega_{\pi}^{2}}{m_{\sigma}^{2}}\left(\frac{n_{B}\left(\omega_{\pi}\right)}{\omega_{\pi}}-\frac{n_{B}\left(\omega_{\sigma}\right)}{\omega_{\sigma}}\right)\right], \\
a_{F}=N_{c} g^{2} \int \frac{d^{3} p}{(2 \pi)^{3}} \frac{n_{F}\left(\omega_{F}\right)}{p^{2} \omega_{F}} \\
b_{F}=-N_{c} g^{2} \int \frac{d^{3} p}{(2 \pi)^{3}} \frac{m_{F}^{2}}{p^{2}} \frac{n_{F}\left(\omega_{F}\right)}{\omega_{F}^{3}} .
\end{gathered}
$$

The subscripts $B$ and $F$ denote the contributions of the boson and fermion loops, respectively. The sign of the fermion contribution in the last equation is crucial. The pion velocity $v$ given by Eq. (14) will become larger than unity when $b<0$, i.e., when the negative fermionic part exceeds the positive bosonic part.

The results (20) and (21) with (22) -(25) can easily be extended to include the case of a hadronic fluid at $T=0$ and $\mu=0$ in $3+1$ dimensional space-time with one spatial dimension compactified to the size $L \equiv \beta$. In this case, fermions may be chosen to be periodic or antiperiodic in the compact dimension. Clearly, the pion velocity in the subspace orthogonal to the compact dimension is equal to the velocity of light. However, velocity parallel to the compact direction is given by

$$
v_{\|}^{2}=1+\frac{b^{\prime}}{a^{\prime}},
$$


where $a^{\prime}=a$ and $b^{\prime}=b$ for antiperiodic and

$$
\begin{gathered}
a^{\prime}=1+a_{B}-\bar{a}_{F}, \\
b^{\prime}=b_{B}-\bar{b}_{F},
\end{gathered}
$$

for periodic fermions, with

$$
\begin{gathered}
\bar{a}_{F}=2 N_{c} g^{2} \int \frac{d^{3} p}{(2 \pi)^{3}} \frac{n_{B}\left(\omega_{F}\right)}{p^{2} \omega_{F}}, \\
\bar{b}_{F}=-2 N_{c} g^{2} \int \frac{d^{3} p}{(2 \pi)^{3}} \frac{m_{F}^{2}}{p^{2}} \frac{n_{B}\left(\omega_{F}\right)}{\omega_{F}^{3}} .
\end{gathered}
$$

Hence, for antiperiodic fermions, the velocity $v_{\|}$given by (26) precisely equals the inverse $v$ calculated at $T=1 / L, \mu=0$. For periodic fermions, the sign of the fermion loop is reversed and the Fermi-Dirac is replaced by a Bose-Einstein distribution.

\section{Results and discussion}

In order to proceed with a quantitative analysis, we have to choose the input parameters from particle physics phenomenology. For the constituent quark mass we take $m_{F}=340$ $\mathrm{MeV}$. The coupling $g$ is then fixed by (3) and (6) $g=m_{F} / f_{\pi}$. The Particle Data Group gives a rather wide range 400-1200 MeV [13] of the sigma meson masses. We shall shortly see that the analysis of the $T=0, \mu \neq 0$ case further restricts this available range.

At $T=0$ the extremum condition (8) for nonnegative $\mu$ and $N_{c}=3$ reads

$$
\sigma^{2}=f_{\pi}^{2}-\frac{3 g^{2}}{\lambda \pi^{2}} \vartheta(\mu-g \sigma)\left[\mu \sqrt{\mu^{2}-g^{2} \sigma^{2}}+g^{2} \sigma^{2} \ln \frac{g \sigma}{\mu+\sqrt{\mu^{2}-g^{2} \sigma^{2}}}\right],
$$

which determines the extremum of the thermodynamic potential

$$
\begin{aligned}
\Omega(\sigma, \mu)= & \frac{\lambda}{4} \sigma^{4}-\frac{\lambda}{2} f_{\pi}^{2} \sigma^{2} \\
& -\frac{\vartheta(\mu-g \sigma)}{(2 \pi)^{2}}\left[\mu \sqrt{\mu^{2}-g^{2} \sigma^{2}}\left(2 \mu^{2}-5 g^{2} \sigma^{2}\right)+3 g^{4} \sigma^{4} \ln \frac{\mu+\sqrt{\mu^{2}-g^{2} \sigma^{2}}}{g \sigma}\right] .
\end{aligned}
$$

The chemical potential $\mu_{c}$ at which chiral symmetry gets restored is given by the condition $\Omega\left(0, \mu_{c}\right)=\Omega\left(\sigma, \mu_{c}\right)$. For $\mu_{c}<g f_{\pi}$, the solution of (31) is $\sigma=f_{\pi}$, so (31) leads to

$$
\mu_{c}=\left(\frac{\lambda \pi^{2}}{2}\right)^{1 / 4} f_{\pi}
$$

Since $\mu_{c}$ is the threshold where extended nuclear matter forms, a reasonable assumption, confirmed by the strong-coupling QCD analysis [14, 15], is

$$
\mu_{c} \leq m_{F}
$$



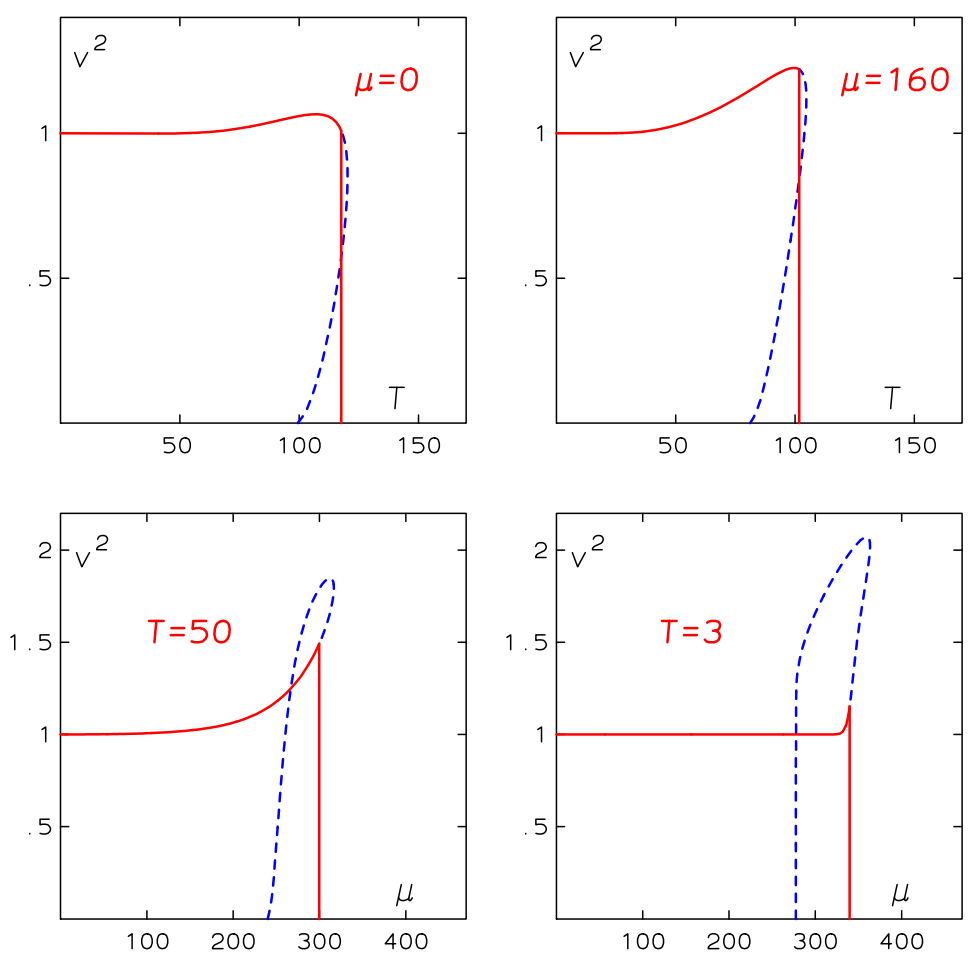

Figure 1: Pion velocity squared as a function of temperature and chemical potential. The dashed line corresponds to a thermodynamically unstable solution. $T$ and $\mu$ are in $\mathrm{MeV}$.

It may be easily verified that with this assumption the pion velocity is constant and equal to light velocity up to the transition point $\mu_{c}$, where it drops to zero.

It is remarkable that Eq. (34) yields an upper bound on the sigma mass

$$
m_{\sigma} \leq \frac{2 m_{F}^{2}}{\pi f_{\pi}} .
$$

If we take $f_{\pi}=92.4 \mathrm{MeV}, m_{F}=340 \mathrm{MeV}$, and saturate the bound, we find $m_{\sigma}=796.5$ $\mathrm{MeV}$. In the following numerical analysis we take this value as an input parameter. Any other choice below this value would not alter our qualitative picture.

Next we analyze the case of nonzero temperature. In Fig. 1 we plot the pion velocity $v$ as a function of temperature for various fixed $\mu$ (upper plots) and as a function of chemical potential for various fixed $T$ (lower plots). For each $T$ and $\mu$ the chiral condensate $\sigma$ is calculated by solving Eq. (8) numerically. The dashed line represents the velocity obtained with thermodynamically unstable solutions for $\sigma$. The actual phase transition takes place at the point $T_{c}$ (for a given $\mu=\mu_{c}$ ) or at $\mu_{c}$ (for a given $T=T_{c}$ ) where the two minima of the thermodynamic potential at $\sigma=0$ and $\sigma\left(T_{c}, \mu_{c}\right)$ are leveled [11]. At the critical point, the pion velocity drops abruptly to zero. We note that there is always a region of temperatures or chemical potentials below the critical point where the pion velocity becomes superluminal. 


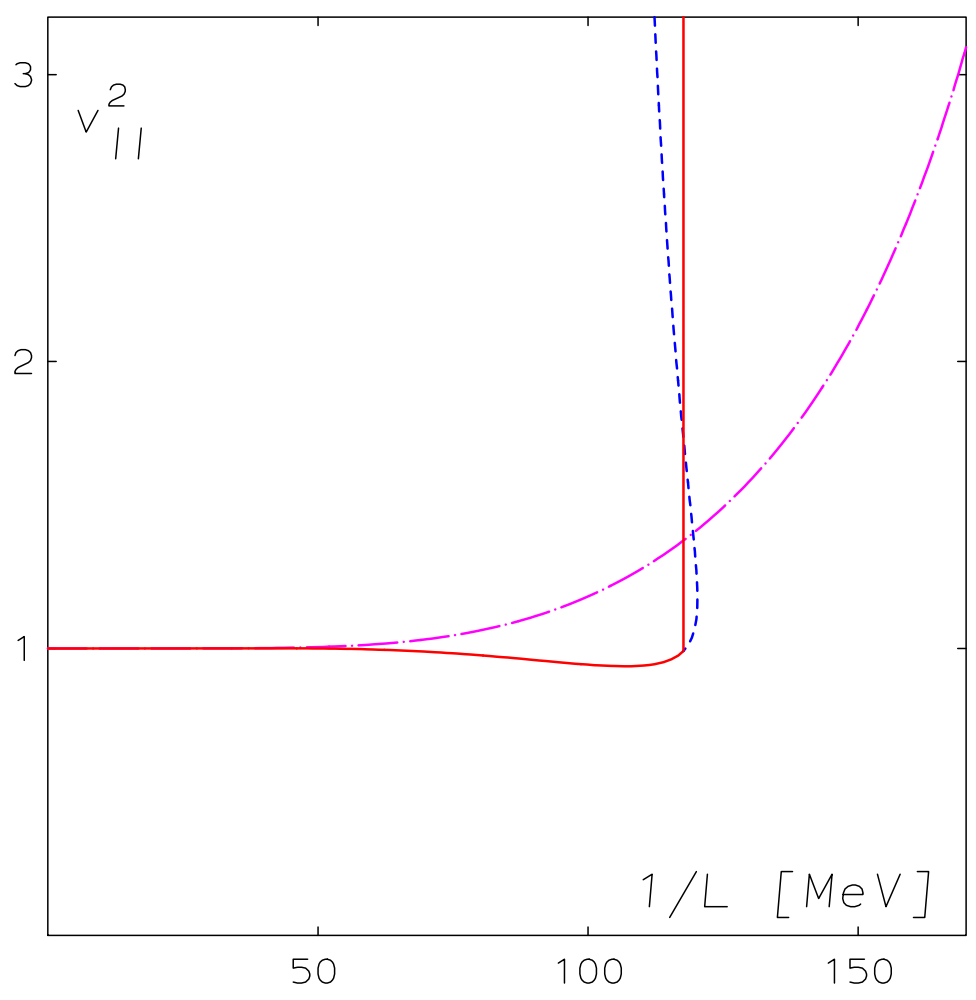

Figure 2: Pion velocity squared as a function of inverse compactification length with antiperiodic (solid line) and periodic (dot-dashed line) fermions. The dashed line corresponds to a physically unstable solution.

Next we discuss the case $T=0$ and $\mu=0$ with one spatial dimension compactified to the size $L \equiv \beta$. The pion velocity $v_{\|}$parallel to the compact direction is plotted in Fig. 2 for both periodic and antiperiodic fermions. In the case of antiperiodic fermions, the compactification size plays the role of inverse temperature and chiral symmetry gets restored at the critical size $L_{c}=1 / T_{c}$. In this case, the velocity plot is just the inverse of the first plot in Fig. 1 .

For periodic fermions, we find no restoration of chiral symmetry, the minimum of the thermodinamical potential corresponding to a nonzero $\sigma$ is always below the minimum corresponding to $\sigma=0$. Owing to the opposite sign of the fermion contribution the velocity $v_{\|}$is always superluminal, monotonously approaching the velocity of light as the size of the compactification $L$ approaches infinity.

\section{$5 \quad$ Summary and conclusion}

We have analyzed the propagation of massless pions at nonzero temperature and nonzero finite baryon density in a sigma model with two quark flavors. By calculating the pion dispersion relation at one-loop order we have shown that pions in the presence of fermions 
become superluminal in a certain range of temperatures and baryon chemical potential.

Furthermore, we have studied the case when one of the spatial dimensions is compactified with fermions obeying periodic or antiperiodic boundary conditions. Restricting attention to $T=\mu=0$, we have calculated the pion velocity $v_{\|}$along the compact direction. We have found that for antiperiodic fermions, pions will propagate superluminally or subluminally, depending on the size of the compact dimension. With periodic fermions, the velocity $v_{\|}$is always larger than the velocity of light.

A superluminal propagation of massless particles may naively seem to contradict special relativity and Lorentz invariance. The most disturbing consequence of a superluminal propagation would be an apparent violation of causality. However, it has been convincingly argued that superluminal effects realized in quantum field theories with a nontrivial vacuum do not lead to causal paradoxes [16. The basic argument goes as follows: once the conditions that determine the vacuum fluctuations are fixed, the propagation velocity in a given reference frame is unique. This implies that it is not possible to send signals both forwards and backwards in time within one reference frame. In other words, the causal loops are forbidden.

It is important to bear in mind that we have considered an idealized situation when chiral symmetry of the original Lagrangian is exact and pions are exactly massless in the broken

symmetry phase. In reality, chiral symmetry is explicitly broken owing to nonvanishing current quark masses. As a consequence, pions become massive even in the spontaneously broken symmetry phase and always propagate slower than light.

\section{Acknowledgments}

We thank I. Dadić for valuable discussions. This work is supported by the Ministry of Science and Technology of the Republic of Croatia under Contract No. 0098002.

\section{References}

[1] M. Gell-Mann and M. Lévy, Nuovo Cimento 16 (1960) 705

[2] R. D. Pisarski and M. Tytgat, Phys. Rev. D54 (1996) R2989

[3] D. T. Son and M.A. Stephanov, Phys. Rev. Lett. 88 (2002) 202302

[4] D. T. Son and M.A. Stephanov, Phys. Rev. D66 (2002) 076011

[5] K. Scharnhorst, Phys. Lett. B236 (1990) 354

[6] J. I. Latorre, P. Pascual, and R. Tarrach, Nucl. Phys. B437 (1995) 60

[7] E. J. Ferrer, V. de la Incera, and A. Romeo, Phys. Lett. B515 (2001) 341

[8] S. Contreras and M. Loewe, Int. J. Mod. Phys. A5 (1990) 2297

[9] A. Gocksch, Phys. Rev. Lett. 67 (1991) 1701 
[10] R. Baier, M. Dirks, and O. Kober, Phys. Rev. D54 (1996) 2222

[11] N. Bilić and H. Nikolić, Eur. Phys. J. C6 (1999) 515

[12] H. Meyer-Ortmanns and B.-J. Schaefer, Phys. Rev. D53 (1996) 6586

[13] K. Hagiwara et al., Review of Particle Physics, Phys. Rev. D66 (2002) 010001

[14] N. Bilić, K. Demeterfi, and B. Petersson, Nucl. Phys. B377 (1992) 651

[15] N. Bilić, F. Karsch, and K. Redlich, Phys. Rev. D45 (1992) 3228

[16] S. Liberati, S. Sonego, and M. Visser, Ann. Phys. 298 (2002) 167 